P. J. J. van Genderen · J. J. Michiels

S. C. P. A. M. van der Poel-van de Luytgaarde

H. H. D. M. van Vliet

\title{
Acquired von Willebrand disease as a cause of recurrent mucocutaneous bleeding in primary thrombocythemia: relationship with platelet count
}

Received 2 March 1994 / Accepted 20 May 1994

Summary We present a 4-year follow-up of a 42-yearold patient with primary thrombocythemia whose clinical course was complicated by two major mucocutaneous bleeding episodes. On both occasions an acquired functional von Willebrand factor deficiency was demonstrated. In contrast to what is reported in the literature, an inverse relationship between platelet number and plasma high-molecular-weight multimers of von Willebrand factor was established.

Key words Primary thrombocythemia Myeloproliferative disorder · von Willebrand factor Acquired von Willebrand disease . Platelets Bleeding

\section{Introduction}

Primary thrombocythemia (PT), a clonal myeloproliferative disorder characterized by a persistent elevation of the platelet count, paradoxically predisposes to both thrombosis and hemorrhages $[13,15]$. Despite the identification of a broad array of specific structural, biochemical, and metabolic platelet defects in patients with PT, a convincing correlation between these defects and the clinical complications of thrombosis and bleeding has never been established $[13,15]$.

Several authors have described a decrease or absence of plasma high-molecular-weight multimers of von Willebrand factor (vWF) in patients with myeloproliferative disorders and associated bleeding tendency $[4,5,10]$. We present here a 4-year follow-up of a patient with PT whose clinical course was complicated by two bleeding episodes associated with an acquired

P. J. J. van Genderen (两) · J. J. Michiels

S. C. P. A. M. van der Poel-van de Luytgaarde

H. H. D. M. van Vliet

Department of Hematology, University Hospital Dijkzigt,

Erasmus University, Dr. Molewater plein 40,

NL-3015 GD Rotterdam, The Netherlands von Willebrand factor deficiency. An inverse relationship between platelet number and plasma high-molecular-weight multimers of von Willebrand factor was demonstrated.

\section{Case report}

A 42-year-old woman was referred to our department in December 1989 with upper abdominal complaints due to massive splenomegaly. Ultrasound examination of the abdomen revealed an enlarged spleen of $20 \mathrm{~cm}$ and thrombosis of the splenic vein. Subsequent endoscopy showed esophageal varices. A slight thrombocytosis of $431 \times 10^{9}$ platelets per liter was established (Fig. 1). Hemoglobin levels, white cell count and differentiation, creatinine, urea, and liver function tests were all normal. Extended hemostatic analysis excluded the presence of a lupus anticoagulant antibody and a deficiency of antithrombin III, protein C, and protein $\mathrm{S}$. Bone marrow smear and biopsy material showed an increased cellularity of normal hematopoiesis, a pronounced increase of clustered megakaryocytes, and the presence of fine reticulin fibers, but the absence of collagen fibers and osteosclerosis, compatible with the diagnosis of PT [15]. The score for the leukocyte alkaline phosphatase stain was 129 (normal 20-100 U/ml). Splenectomy in December 1989 (spleen weight $788 \mathrm{~g}$ ) was uneventful. In March 1990, the platelet count rose to about $3500 \times 10^{9} / 1$; this was associated with spontaneous mucocutaneous bleeding consisting of easy bruising, recurrent ecchymoses after minor injuries, and melena, causing a fall of the hemoglobin level from 7.3 to 4.6 $\mathrm{mmol} / 1$. Extended coagulation tests revealed a functional deficiency of plasma von Willebrand factor [von Willebrand factor antigen (vWF:Ag) $1.36 \mathrm{U} / \mathrm{ml}$; ristocetin co-factor activity (vWF:RCoF) $0.40 \mathrm{U} / \mathrm{ml}$, and collagen binding activity (vWF: CBA) $0.22 \mathrm{U} / \mathrm{ml}$, normal values for all vWF-related activities $0.60-1.40 \mathrm{U} / \mathrm{ml})$ ] and a prolonged Ivy bleeding time $(>15$ min; normal $\leq 4 \mathrm{~min}$ ). Platelet aggregation tests were normal.

From that time on we prospectively studied plasma von Willebrand factor activities in relation to the platelet count. Reduction of the platelet count with hydroxyurea resulted in disappearance of the bleeding symptoms and the functional von Willebrand factor defect and in a normalization of the Ivy bleeding time. At platelet counts between 700 and $1650 \times 10^{9} / 1$ for more than 1 year neither the bleeding nor the functional von Willebrand factor defect (vWF: Ag varied from 1.22 to $2.00 \mathrm{U} / \mathrm{ml}$; vWF: RCoF from 0.64 to $1.72 \mathrm{U} / \mathrm{ml}$, and $\mathrm{vWF}$ : $\mathrm{CBA}$ from 0.67 to $1.26 \mathrm{U} / \mathrm{ml}$ ) recurred.

Discontinuation of hydroxyurea because of side effects (headache and nausea) in August 1992 resulted in a rapid increase in 


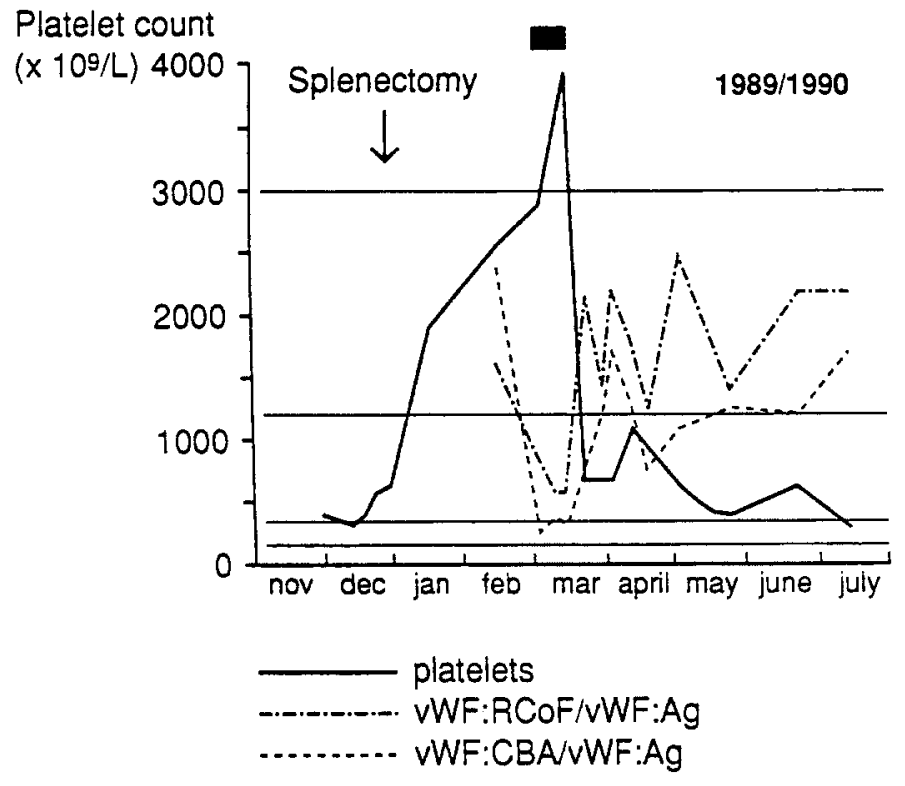

Fig. 1 The clinical course of a patient with primary thrombocythemia was complicated by two mucocutaneous bleeding episodes at platelet counts above $2000 \times 10^{9} / 1$. On both occasions a functional von Willebrand factor deficiency was demonstrated; this disappeared after reduction of the platelet count with hydroxyurea. Von Willebrand factor activities are expressed as the ratio of either vWF:RCoF/vWF:Ag or vWF:CBA/vWF:Ag to denote the amount of functionally active von Willebrand factor. The upper two horizontal lines represent the normal range for the vWF: RCoF/vWF:Ag and vWF:CBA/vWF:Ag ratios. The lower two horizontal lines indicate the normal range for the platelet count

circulating platelets (platelet counts $3700-4000 \times 10^{9} / 1$ ) and recurrence of the mucocutaneous bleeding tendency, which was characterized by easy bruising, prolonged bleeding after minor cuts, and menorrhagia, causing a fall of the hemoglobin level from 7.0 to $3.7 \mathrm{mmol} / \mathrm{l}$. Again, a functional von Willebrand factor defect (vWF:Ag $1.31 \mathrm{U} / \mathrm{ml}$; vWF: RCoF $0.46 \mathrm{U} / \mathrm{ml}$, and vWF: CBA 0.11 $\mathrm{U} / \mathrm{ml}$ ) and a prolongation of the bleeding time $(>15 \mathrm{~min})$ were demonstrated. The bleeding symptoms and the functional von Willebrand factor defect disappeared after reduction of the platelet count with hydroxyurea. The hemoglobin level completely recovered with oral iron administration.

\section{Material and methods}

All hemostatic tests were performed using routine procedures. Platelets were counted electronically in blood anticoagulated with EDTA. Bleeding times were measured according to Ivy et al. [9]. Von Willebrand factor antigen was assayed by an ELISA using rabbit anti-human vWF and rabbit horseradish peroxidase-conjugated anti-human vWF polyclonal antibodies [7]. Ristocetin cofactor activity was assayed with formalin-fixed platelets [11] (intra-assay variation $10 \%$; inter-assay variation $11 \%$ ). The binding of vWF to collagen was measured according to the ELISA-based method of Brown and Bosak [3], with slight modifications (intraassay variation $5 \%$, inter-assay variation $9 \%$ ). Microtiter plate wells (A/S Nunc, Roskilde, Denmark) were coated overnight at $4^{\circ} \mathrm{C}$ with $100 \mu \mathrm{l}$ of a $0.2-\mathrm{mg} / \mathrm{ml}$ suspension of collagen (bovine achilles tendon, type I, Sigma, St. Louis, USA) in $20 \mathrm{~m} M$ acetic acid. After washing with PBS-Tween, $100 \mu l$ of a 1/40 dilution of the test plasmas in PBS-Tween-albumin were added to the wells and incubated for $2 \mathrm{~h}$ at room temperature. After rinsing, the

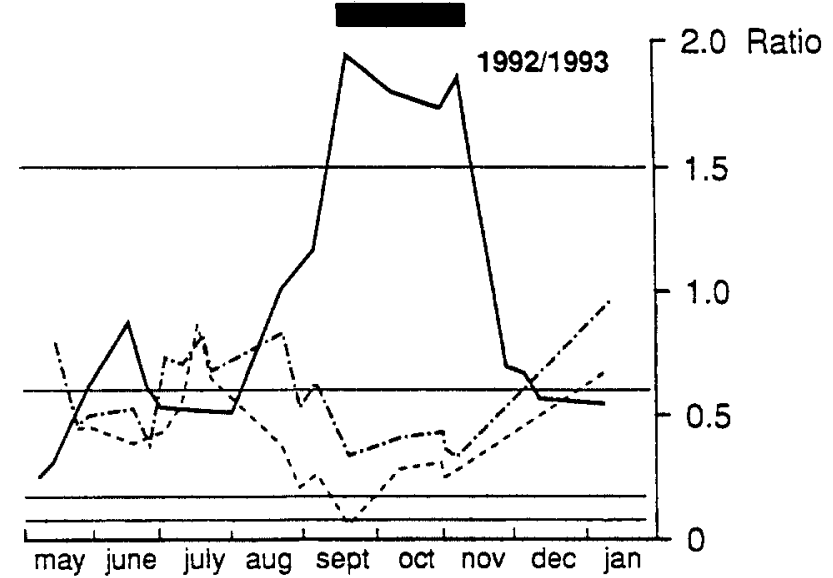

wells were incubated with $1 / 200$ dilution in PBS-Tween-albumin of horseradish peroxidase-conjugated rabbit polyclonal immunoglobulins to human vWF (Dakopatts, Denmark) for another $2 \mathrm{~h}$ at room temperature. After washing, $100 \mu \mathrm{l}$ ABTS substrate solution was added. After $15 \mathrm{~min}$ incubation the color development was stopped by the addition of $10 \mu l$ concentrated acetic acid and the extinction was measured at $414 \mathrm{~nm}$. The multimeric pattern of vWF was visualized according to Brosstad et al. [2].

The relationship between platelet count and plasma levels of von Willebrand factor was analyzed by linear regression and Spearman's rank correlation test. Statistical significance was accepted at $p<0.05$.

\section{Results}

The clinical course of the described patient was complicated by two major bleeding events. On both occasions an acquired von Willebrand defect and a prolonged Ivy bleeding time were demonstrated, occurring at platelet counts above $2000 \times 10^{9} / 1$ (Fig. 1). At times of manifest bleeding, the $\mathrm{vWF}: \mathrm{RCoF} / \mathrm{vWF}: \mathrm{Ag}$ ratios and vWF: $\mathrm{CBA} / \mathrm{vWF}: \mathrm{Ag}$ ratios were decreased at $0.40 \pm 0.13$ and $0.19 \pm 0.08$, respectively, indicating a functional deficiency of VWF. Reduction of the platelet count with hydroxyurea resulted in disappearance of the bleeding symptoms as well as of the acquired functional von Willebrand defect (Fig. 1) and in a normalization of the Ivy bleeding time. A significant inverse relationship between the platelet count and the ratios of vWF: RCoF/vWF: $\mathrm{Ag}(r=-0.728, p<0.001)$ and vWF:CBA/vWF:Ag $(r=-0.834, p<0.001)$ was demonstrated (Fig. 2). Analysis of the vWF multimers at different levels of thrombocytosis (Fig. 3) revealed a progressive decrease of the high-molecular-weight multimers of vWF with increasing platelet counts. 


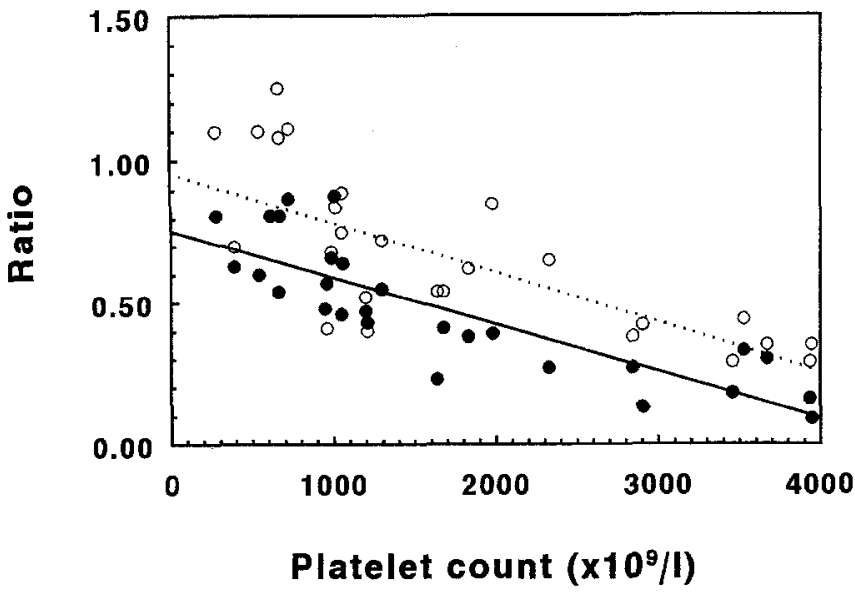

Fig. 2 Intrapatient relationship between platelet count and either $\mathrm{vWF}: \mathrm{RCoF} / \mathrm{vWF}: \mathrm{Ag}$ ratio (open symbols) or vWF:CBA/ VWF: Ag ratio (closed symbols) and corresponding linear curve fits (broken and solid lines, respectively)

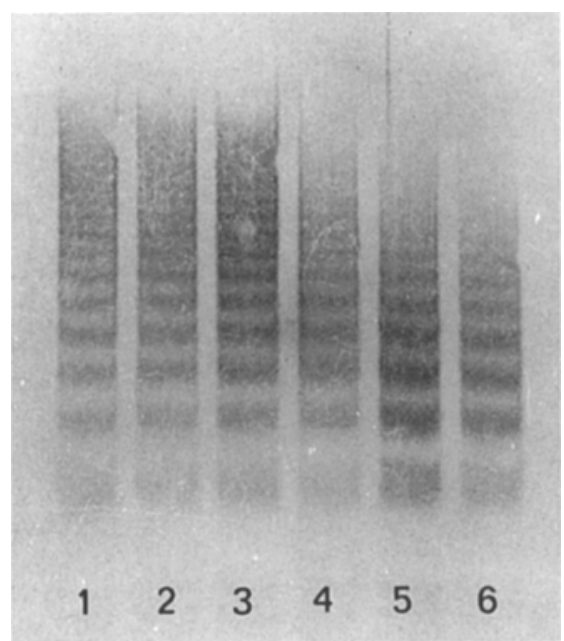

Fig. 3 Multimeric pattern of plasma von Willebrand factor of normal pooled plasma and patient's plasma at different levels of thrombocytosis. Lane 1, normal pooled plasma; lane 2-6, multimeric pattern of plasma vWF of the patient at platelet 4 counts of $390 \times 10^{9} / 1,727 \times 10^{9} / 1,1058 \times 10^{9} / 1,2905 \times 10^{9} / 1$, and $3938 \times 10^{9} / 1$, respectively. Note the progressive decrease of high-molecularweight multimers of von Willebrand factor in the plasma of the patient with increasing platelet counts

\section{Discussion}

In the reported patients with PT and an acquired von Willebrand factor deficiency, bleeding usually became apparent at platelet counts in excess of $1000 \times 10^{9} / 1$ and disappeared or improved after reduction of the platelet count to below $1000 \times 10^{9} / 1$, suggesting that platelets play a pivotal role in the etiology of such bleeding $[4,5$, 10]. However, a clear relationship between platelet count and clinical complication of bleeding has never been established $[13,15]$.

In the patient presented here an inverse relationship between platelet number and plasma vWF was demon- strated, resulting in a functional von Willebrand factor defect at platelet counts above $1000 \times 10^{9} / 1$. With increasing platelet counts both the $\mathrm{vWF}: \mathrm{RCoF} / \mathrm{vWF}: \mathrm{Ag}$ and $\mathrm{vWF}: \mathrm{CBA} / \mathrm{vWF}: \mathrm{Ag}$ ratios progressively decreased, suggesting a deficiency of the high-molecularweight multimers of vWF, the most potent forms of vWF in securing hemostasis. The decrease of high-molecular-weight multimers of vWF at platelet counts above $1000 \times 10^{9} / 1$ was also demonstrated by visualization of the multimeric distribution of $\mathrm{vWF}$. However, clinical bleeding became manifest only at platelet counts in excess of $2000 \times 10^{9} / 1$. Laboratory investigations at times of bleeding showed a deficiency of vWF:CBA activity, in contrast to only subnormal values of vWF:RCoF activity, suggesting that the vWF: CBA activity is a more reliable parameter of in vivo $\mathrm{vWF}$ function than the ristocetin co-factor activity.

This report and previous studies $[4,5,10]$ indicate that cytoreduction of the platelet count below $1000 \times 10^{9} / 1$ favors prevention of bleeding complications in PT, which is consistent with recent epidemiological findings that hemorrhages in PT occur more frequently at platelet counts in excess of $1000 \times 10^{9} / 1[1,8$, $15,16]$. However, in patients with reactive thrombocytosis hemorrhagic complications occur rarely or not at all, which suggests that more than a rise in the number of circulating platelets is needed to explain the observed hemorrhagic predisposition in primary thrombocythemia $[13,15]$.

The pathogenesis of the von Willebrand factor deficiency in PT is unclear. It has been demonstrated that the deficiency of the high-molecular-weight multimers of $\mathrm{VWF}$ is a phenomenon occurring in vivo and is not due to proteolysis in vitro $[5,14]$. The observed increase in proteolytic cleavage fragments of the vWF subunit in PT might be attributed to in vivo degradation of von Willebrand factor by platelet-derived calcium-activated neutral proteases (calpains) and human leukocyte elastase [5]. However, others suggest that the decrease of the high-molecular-weight multimers of VWF is the result of binding of vWF to an increased number of circulating activated platelets at high platelet counts $[12,14]$. Although the exact mechanism operative in PT remains to be determined, our data imply that the decrease of the high-molecular-weight multimers of von Willebrand factor is related to an elevated number of circulating platelets. Interestingly, Budde and colleagues [6] have recently shown that the decrease of the high-molecular-weight multimers of von Willebrand factor is also demonstrable in the plasma of patients with reactive thrombocytosis. Therefore, an elevated number of circulating platelets is more likely to be responsible for the observed decrease in high-molecular-weight multimers of von Willebrand factor than the presence of presumed functionally abnormal platelets derived from clonal proliferation. However, further studies of larger patient groups are indicated to clarify the correlation of vWF abnormalities and the occur- 
rence of a bleeding tendency in the course of myeloproliferative disorders, as is evident from the presented case, whereas such a correlation has not been documented in patients with reactive thrombocytosis.

Acknowledgement We wish to thank dr P. J. J. Leeuwerik (Lievensberg Hospital, Bergen op Zoom) for clinical information and referral of the patient.

\section{References}

1. Bellucci S, Janvier M, Tobelem G, Flandrin G, Charpak Y, Berger R, Boiron M (1986) Essential thrombocythemias: clinical, evolutionary and biological data. Cancer 58:2440-2447

2. Brosstad F, Kjonniksen I, Ronning B, Stormorken H (1986) Visualization of von Willebrand factor multimers by enzymeconjugated secondary antibodies. Thromb Haemost 55:276278

3. Brown JE, Bosak JO (1986) An ELISA test for the binding of von Willebrand factor antigen to collagen. Thromb Res 43:303-311

4. Budde U, Schaefer G, Mueller N, Egli H, Ruggeri ZM, Zimmerman TS (1984) Acquired von Willebrand's disease in the myeloproliferative syndrome. Blood 64:981-985

5. Budde U, Dent JA, Berkowitz SD, Ruggeri ZM, Zimmerman TS (1986) Subunit composition of plasma von Willebrand factor in patients with the myeloproliferative syndrome. Blood $68: 1213-1217$

6. Budde U, Scharf RE, Franke P, Hartmann-Budde K, Dent J, Ruggeri ZM (1993) Elevated platelet count as a cause of abnormal von Willebrand factor multimer distribution in plasma. Blood 82:1749-1757
7. Cejka J (1984) Enzyme immunoassay for factor VIII-related antigen. Clin Chem 28:1356-1358

8. Fenaux P, Simon M, Caulier MT, Lai JL, Goudemand J (1990) Clinical course of essential thrombocythemia in 147 cases. Cancer 66:549-556

9. Ivy AC, Nelson D, Bucher G (1941) The standardization of certain factors in the cutaneous 'venostasis' bleeding time technique. J Lab Clin Med 26:1812-1815

10. Lopez-Fernandez MF, Lopez-Berges C, Martin R, Pardo A Ramos FJ, Battle J (1987) Abnormal structure of von Willebrand factor in myeloproliferative syndrome is associated to either thrombotic or bleeding diathesis. Thromb Haemost 58:753-757

11. Macfarlane DE, Stibbe J, Kirby EP, Zucker MB, Grant RA McPherson J (1975) A method for assaying von Willebrand factor (ristocetin cofactor). Thromb Diath Haemorrh 34:306308

12. Sato K (1988) Plasma von Willebrand factor abnormalities in patients with essential thrombocythemia. Keio J Med 37:54 71

13. Schafer AL (1991) Essential thrombocythemia. Prog Thromb Hemost 10:69-96

14. Tatewaki W, Takahashi H, Takakuwa E, Wada K, Shibata A (1989) Plasma von Willebrand factor proteolysis in patients with chronic myeloproliferative disorders: no possibility of ex vivo degradation by calcium-dependent proteases. Thromb Res 56:191-199

15. van Genderen PJJ, Michiels JJ (1993) Primary thrombocythemia: diagnosis, clinical manifestations and management. Ann Hematol 67:57-62

16. van Genderen PJJ, Michiels JJ (1994) Erythromelalgic thrombotic and haemorrhagic manifestations of thrombocythaemia. Presse Med 23:73-77 